\title{
A Novel Modulation Scheme of 8X8 MIMO in Industry 4.0
}

\author{
Rajashree Suryawanshi, Sathyabama Institute of Science and Technology, Semmancheri, India \\ P. Kavipriya, Sathyabama Institute of Science and Technology, Semmancheri, India \\ B.P. Patil, Army Institute of Technology, Pune, India \\ D https://orcid.org/0000-0001-7369-0645
}

\begin{abstract}
A key component in the usage of Industry 4.0 arrangements is the up and coming age of system network, LTE/LTE advanced today, and 5G, later on. Industry 4.0 is conveying private LTE arranges today, as they trust it will give them an upper hand and a solid head start when 5G opens up. LTE uses multiple input multiple output (MIMO), a promising technique to accomplish the necessary data rate. This article presents consequences of $8 \times 8$ MIMO framework in fading channel utilizing V-BLAST by means of zero-forcing (ZF) with standard ZF. The relative investigation in the fading channel on account of symbol error proves V-BLAST by means of ZF has predominant execution than standard ZF finder. In addition to this, in order to support adaptive modulation, various M-QAM modulation schemes for these detectors are compared. This comparison shows 128-QAM performs superiorly to staying two. This investigation is carried out to help utilization of MIMO in LTE to support Industry 4.0.
\end{abstract}

\section{KEYWORDS}

Industry 4.0, LTE, MIMO, V-BLAST, ZF

\section{INTRODUCTION}

Industry 4.0 gives computerized change in the physical framework we use to reasonably extricate assets, move them to showcase, make, power, work what's more, administration all parts of our mechanical world, including the administration of our urban areas and the prosperity of our residents. Industry 4.0 empowers enterprises to intertwine physical with advanced procedures by interfacing all sensors, machines and laborers in the most adaptable way accessible. The major requirement of Industry 4.0 is large bandwidth. Industry 4.0 uses Long Term Evaluation (LTE) as the important factor. LTE uses MIMO to fulfill the requirement of peak data rate as described by Wei et al. (2007) and bandwidth. So as to improve information rate and channel limit different radio wires at the source region as well as at the beneficiary region are utilized as described by Telatar (1999). Here to understand and get the design ideas of MIMO system as described by Paulraj et al. (2001), practical MIMO systems along with its performance and limitations are studied as described by Nabar et al. (2004). The work of Golden et al. (1999) towards transmitting end, de-multiplexing of moving toward data stream in 
'n' self-facilitated accomplice streams is completed and transmits through accepting wires. Getting reception apparatuses will get the uproarious sign. The work of Wolniosky et al. (1998) recognition happens with three principle tasks: Interference nulling, ideal requesting and impedance is crossing out. Images with most elevated sign to commotion proportion (SNR) are recognized by utilizing ideal requesting. Along these lines, incorporation of direct calculation and non-straight calculation is done with ' $\mathrm{M}$ ' transmitting reception apparatuses and ' $\mathrm{N}$ ' getting radio wires separately in Ricean level blurring divert into BLAST as described by Nabar et al. (2005). This article considers, accepting in receipt of wires $(\mathrm{N})$ be viewed as more otherwise equivalent in number to that of transmitting reception apparatuses $(\mathrm{M})$, the sub stream which is recognized first having decent variety addition of $\mathrm{N}-\mathrm{M}+1$ as described by Ginis et al. (2001) as it were. The idea of a large spatial modulation (SM) supported V-BLAST (SM V -BLAST) system, where SM symbols are map to a structure V-BLAST is proposed in as described by Xiao et al. (2018). Here is no comprehensive as well as workable solution to optimize the progressive joint source coding, space time coding as well as channel coding of packets. A parametric move toward solving this type of extremely nonlinear optimization difficulty is applied inside MIMO framework as described by Chang et al. (2020). Network devices with Internet of Things (IoT), equipped with wireless sensor nodes, storing as well as processing power and limited battery capacity. Therefore, offered tools, such as sensing, computing plus communication must be used powerfully throughout the process. It generates the need for an effective routing algorithm. A multiobjective algorithm for routing optimization is suggested in as described by Ganesan R. et al. (2020).

This article gives correlation knowledge on the presentation of 8 X 8 MIMO frameworks in blurring channel for V-BLAST design by methods for Zero Forcing (ZF) finder and standard ZF indicator alongside different regulation plans. Image blunder rate is utilized for correlation of these collectors; V-BLAST by methods for ZF shows noteworthy improvement over standard ZF so as to help versatile regulation. V-BLAST by means for ZF along with standard ZF finders of 8 X 8 uncoded MIMO are looked at in as described by Jalal J. Hamad Ameen (2014) for 4 QAM tweak procedure. So as to help versatile balance this article analyzes M-QAM where M=64, 128 and 256 alongside these two identifiers. Recreation results in performance of 128-QAM is superior to staying two. Industry 4.0 uses LTE or LTE - Advanced till 128-QAM, on this basis evaluation is completed so that it helps the usage of MIMO in LTE.

\section{SYSTEM MODEL}

Multiple antenna technique worn in wireless communication system offers trustworthy transmission as described by Y.L.C et al. (2005), Prasad N. et al. (2001). Different schemes exploit such MIMO system as described by Ilesanmi B. et al. (2013), Shah Shweta et al. (2014). Spatial multiplexing and diversity techniques helps MIMO framework as shown in Figure 1 to carry out required experimentation as described by Volker Kühn (2006), Special issue on complexity reduction in MIMO (2009).

BLAST, V-BLAST Jiang Y. et al. (2005), Loyka S. et al. (2004)and STBC, these techniques are often worn for analysis of MIMO communication. In this article V-BLAST technique is used

Figure 1. MIMO Framework

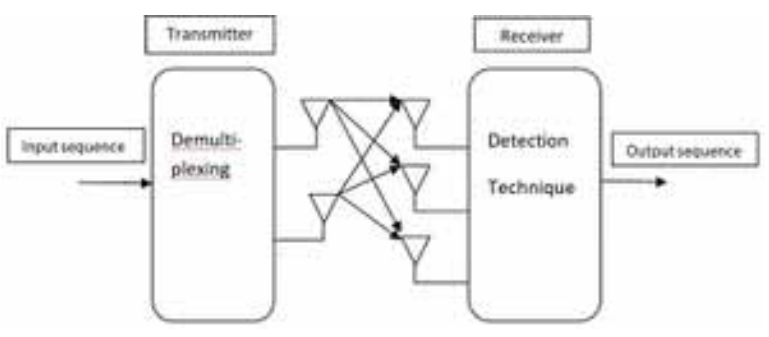


because of its advantages. V-BLAST is also use in spatial multiplexing for transmission of several self-governing data streams on several antennas promptly. This article gives insight on comparison of two linear detectors, standard ZF detector as well as V-BLAST by means of ZF. Some useful signal energy is thrown away by ZF detector during complete interference cancellation process.

As shown in Figure 2 channel from transmitting end $x$ to receiving end $y$ is represented by $h_{x y}$. Transmission of the symbols and impulse response of channels which follows rayleigh distribution are as shown in Table 1 as described by (de Jong \& Willink, 2005).

Here, the transmitter is denoted by $t$ and QAM modulated symbols are $A_{1}, A_{2}, A_{3}, \ldots \ldots ., A_{16}$. The matrix equation $\mathrm{B}=\mathrm{HA}+\mathrm{N}$ is used for representation of received vectors:

- $\mathrm{b}_{\mathrm{x}}=$ Reception of signal at $\mathrm{y}^{\text {th }}$ receiving antenna;

- $\mathrm{n}_{\mathrm{x}}$ is the collective AWGN noise at the $\mathrm{y}^{\text {th }}$ receiving antenna.

In each schedule opening, inclusion of 64 channels is done and the channel motivation reaction as per Rayleigh distribution. where $\mathrm{h}=\mathrm{a}+\mathrm{ib}$; here Gaussian arbitrary factors are, a and $\mathrm{b}$. Initially Channel lattice ( $\mathrm{H}$ framework) is produced and afterward the various modulation schemes adjusted information images (X network) are created.

\section{ZF Algorithm}

$\mathrm{ZF}$ is a linear detection method of usual MIMO detection. In this calculation, the channel lattice obstruction is nulled out by channel reversal with the heaviness of the grid. In wireless communication system multiuser interference signals can be nulled in multiple antenna transmitters by using spatial signal processing which is Null steering or Zero forcing. In ZF required noteworthy response in the

Figure 2. The channel from transmitting end $x$ to receiving end $y$ is represented by $h_{x y}$

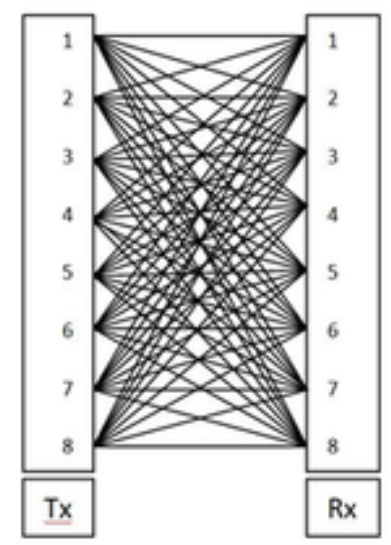

Table 1. Transmission of the symbols and impulse response of channels which follows rayleigh distribution

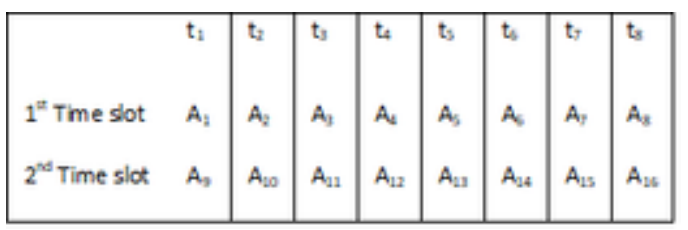


clouds by way of signal to noise ratio (SNR) helps into achieving full multiplexing gain. Multiuser interferences can't be nulled with bars produced by the inaccurate CSIT hence the residue is present.

In standard ZF detector approximation of the transmitter matrix is generated as $\widehat{A}=\operatorname{pinv}(H) B$, inv is pseudo inverse function and $(\hat{A})$ is approximation of $A$. By and by the assessments be send interested in the Maximum Likelihood decoder in addition to the nearby star gathering end be gotten in favor of all of these, autonomously.

\section{V-Blast Algorithm}

V-BLAST broadcast strategy is clarified what's next. De-multiplexing of an information stream in sub stream $\mathrm{M}$ is known like layers. Circular shifting of the layers across $\mathrm{M}$ transmit antennas takes place during every transmission interval in case of diagonal bell laboratories layered space time (D - BLAST). The result with circular shift is a diagonal structure, observed transversely between space as well as time. But in V-BLAST case, these layers are horizontally arranged transversely between space and time and as shown in Figure 3, the removal of cycling operation takes place before transmission. At the receiving end, received signals will be observed by overlay of fading icons $\mathrm{M}$ along with an Additive White Gaussian Noise (AWGN) per antenna it receives. As for both these BLAST systems, arrangement of the layers is different across space and time, be that as it may, for both the frameworks the location procedure for each got vector is performed vertically. The detection process is explained as follows.

\section{Interference Suppression or Nulling Effect}

The received vector is projected on null subspace. Then the suppression operation is performed to null out the interference. Then the ordinary discovery of this primary Image is taken.

\section{Interference Termination or Subtraction Effect}

The straight (obstruction concealment) and nonlinear (sequential abrogation) calculations are joined in BLAST identification calculation. The proliferation of choice blunders happens is the significant disadvantage of BLAST calculations. The effect of the obstruction concealment on the at first recognized images is that they are profited by lower gets assorted variety than later ones. In this way, every image is getting favorable position of inconsistent assorted variety.

Figure 3. The removal of cycling operation that takes place before transmission

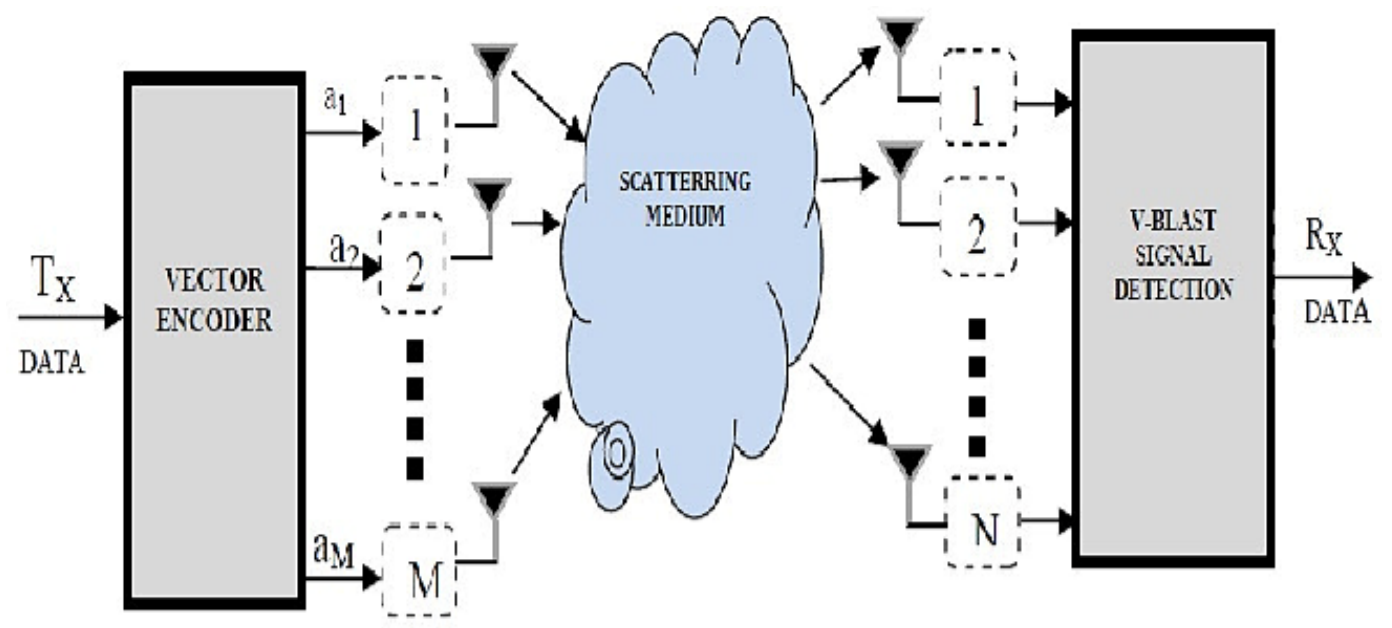


Both V-BLAST as well as D-BLAST is associated with following comparison. In V - BLAST coded otherwise uncoded layers can be used, but D - BLAST uses only coded layers. Spatial diversity of every layer increases especially above gradually fading channels cycling. So cycling is removed in V-BLAST. D-BLAST has diagonal structure but V-BLAST does not have diagonal structure. So each layer of D-BLAST gets benefited from same diversity advantage while the other one gives unequal diversity advantages. For optimum performance, D-BLAST needs inter stream coding methodology. In case of D-BLAST wastage is observed in some space-time at the beginning as well as at the ending of the burst.

\section{Characteristics of V-BLAST by Means of ZF}

In this the transmitted information streams can be isolated by utilizing direct collector front finishes; altogether decreases unraveling intricacy of Maximum likelihood (ML) receiver. Also, after that every one of the streams is decoded autonomously. Basic straight collector has low computational multifaceted nature and experiencing commotion improvement. Be that as it may, at high SNR it works best. In the ZF collector joint deciphering issue are changed over into M single stream disentangling issues. So the recipient multifaceted nature is altogether decreased with this ZF collector. This multifaceted nature decrease advantage is happening at the expense of clamor improvement.

Multiple antenna system is promising to give very high spectral efficiency. V-BLAST system uses multiple antennas for data transmission and these are decoded at the detector. So the nonlinear interference operation is difficult. And V-BLAST performance survey using ZF-SIC detector is hard as it cancels nonlinear interference. V-BLAST can be applied with either minimum mean square error - MAP law or ZF. In this article V - BLAST by means of ZF rule adopted, which one is V BLAST variation drawn as of ZF law. V - BLAST was as a successive method of cancelation using the ZF method.

\section{V-BLAST Algorithm With ZF}

Start

$$
i=1
$$

Recourse

$$
\begin{gathered}
k_{i}=\arg \min \left(W_{i}\right)_{j}^{2} \\
j \in\left\{k_{1}, k_{2}, \ldots, k_{i-1}\right\} \\
b_{k_{i}}=\left(W_{i}\right)_{k_{i}} b_{i} \\
\widehat{A}_{k_{i}}=Q\left(b_{k_{i}}\right) \\
r_{i+1}=r_{i}-A_{k_{i}}(H)_{k_{i}} \\
W_{i+1}=\operatorname{pinv}(H)_{\bar{k}_{i}} \\
i=i+1
\end{gathered}
$$


wherever pinv denote the inverse pseudo operation, $\left(W_{i}\right)_{j}$ is $\mathrm{W}_{i}{ }^{\prime} \mathrm{s}_{j}{ }^{\text {th }}$ line, $\mathrm{Q}$ (.) denotes quantizer at nearest point of constellation, $(\mathrm{H})_{\mathrm{ki}}$ is $\mathrm{k}_{\mathrm{i}}^{\text {th }}$ column of $\mathrm{H}, H_{k i}$ is matrix obtain after column zeroed $\mathrm{k}_{1}$, $\mathrm{k}_{2}, \ldots . \mathrm{k}_{\mathrm{i}}$ of $\mathrm{H}$, in addition to $(H)_{\bar{k}_{i}}$ is inverse rapid pseudo $(H)_{\bar{k}_{i}}$.

\section{Use of Adaptive Modulation}

Adaptive modulation (AM) can be utilized to improve the exhibition of the framework because of the ceaseless change of the remote channel. Here, we expect that every client chooses from three regulation choices of $\mathrm{M}-\mathrm{QAM}$ where $\mathrm{M}=64,128$ as well as 256 dependent on evaluated SER.

\section{RESULT AND DISCUSSION}

In recreation, here the SER of detection calculations is considered, e.g. MMSE, ZF, plus V - BLAST by means of ZF as well as V - BLAST by means of MMSE in favor of 8x8 MIMO-OFDMA frames. To confirm, reenactment is done in MATLAB (2015a). The interpretation is finished using ZF along with V-BLAST strategies with balanced M-QAM sign by means of Rayleigh blurring channel in addition to this outcomes are examined afterwards.

Figure 4 compares SER of ZF as well as V - BLAST MIMO detector for 8x8 uncoded MIMO for $\mathrm{M}-\mathrm{QAM}, \mathrm{M}=64$.

This chart shows that ZF-VBLAST gives better SER execution when contrasted with ZF discovery in $8 \times 8$ uncoded MIMO framework with 64-QAM true to form. Blue line speaks to ZF and red line speaks to ZF-VBLAST.

Results shows that ZF-VBLAST has better SER execution VBLAST, a non-straight identification technique gives better SER when contrasted with direct ZF collector. For SNR of 35db ZFVBLAST shows an improvement of half when contrasted with ZF.

Figure 5 compares SER of ZF as well as V - BLAST MIMO detector for 8x8 uncoded MIMO for $\mathrm{M}$ - QAM, $\mathrm{M}=128$.

Figure 6 compares SER of standard ZF as well as V - BLAST MIMO detector for 8x8 uncoded MIMO for M - QAM, M=256.

Figure 7 compares SER of ZF as well as V - BLAST MIMO detection schemes for $8 \mathrm{x} 8$ uncoded MIMO.

The V - BLAST by means of ZF is compared with standard ZF for adaptive modulation. The results of comparison show the V-BLAST results improved than the standard ZF through terms of symbol error rate. Figure 4, Figure 5 as well as Figure 6 be utilized on behalf of examination of V-BLAST by means of ZF along with standard ZF in support of adaptive modulation with M-QAM where $\mathrm{M}=64,128$ and 256 individually. This demonstrates V-BLAST by means of ZF perform

Figure 4. Compares SER of ZF as well as V - BLAST MIMO detector for $8 \times 8$ uncoded MIMO for M - QAM, M=64

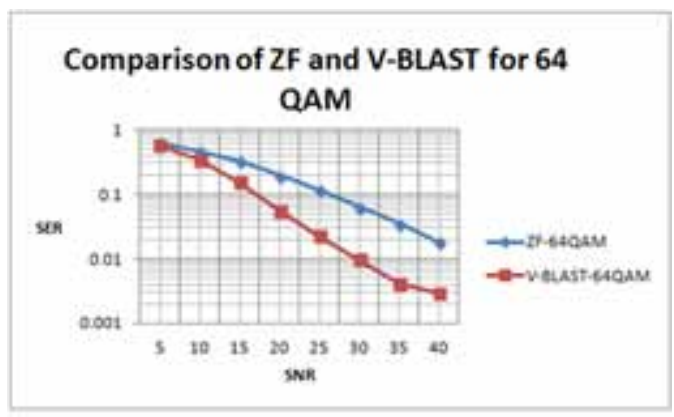


Figure 5. Compares SER of ZF as well as V - BLAST MIMO detector for 8x8 uncoded MIMO for M - QAM, M=128

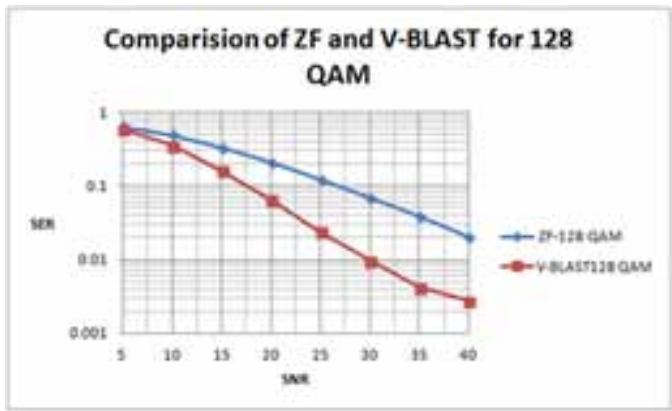

Figure 6. Compares SER of standard ZF as well as V - BLAST MIMO detector for 8x8 uncoded MIMO for M - QAM, M=256

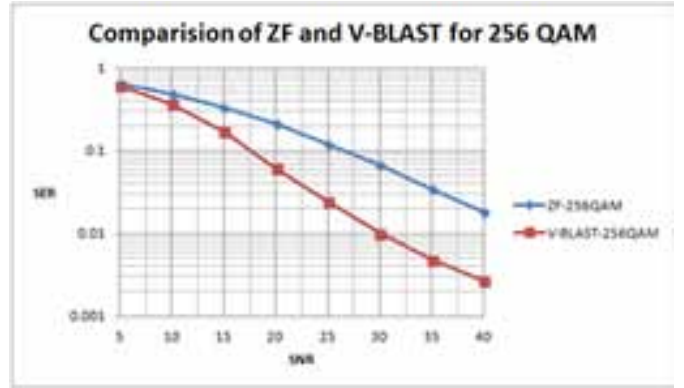

Figure 7. Compares SER of ZF as well as V - BLAST MIMO detection schemes for $8 \times 8$ uncoded MIMO

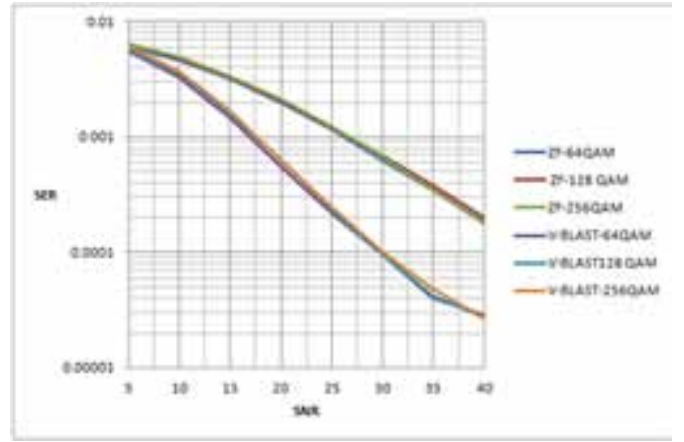

extensively greater now than ever standard ZF. Both those findings are compared into Figure 7 in support of examination of V - BLAST by means of ZF and standard ZF detection on 8 X 8 MIMO uncoded systems by means of adaptive modulation with three modulation schemes of M-QAM where $\mathrm{M}=64,128$ as well as 256 . The simulations show that for the same 30dB SNR in the Rayleigh fading channel, V-BLAST provides SER of 6.93E-03, and the SER of standard ZF detector is 5.44E-02 module with modulation scheme 128-QAM by means of the ZF detector system. At low SNR values 5 - $30 \mathrm{~dB}$ adaptive modulation with $\mathrm{M}=64$ is chosen as it gives less value of SER with both these detectors. At high SNR $31-40 \mathrm{~dB}$ adaptive modulation with $\mathrm{M}=128$ is chosen as it gives less value of SER with both these detectors. The diagram shows closeness between these two frameworks at lower estimation of SNR and gap decreases. 
Table 2. Comparison of detection schemes with type of modulation

\begin{tabular}{|l|l|c|cc|c|c|}
\hline $\begin{array}{l}\text { Detection } \\
\text { Scheme }\end{array}$ & $\begin{array}{l}\text { Modulation } \\
\text { Scheme }\end{array}$ & 5 & 10 & 20 & 31 & 40 \\
\hline V - BLAST & 256-QAM & $6.01 \mathrm{E}-01$ & $2.01 \mathrm{E}-01$ & $3.88 \mathrm{E}-02$ & $8.09 \mathrm{E}-03$ & $2.62 \mathrm{E}-03$ \\
\cline { 2 - 7 } by mean & 128-QAM & $5.76 \mathrm{E}-01$ & $1.94 \mathrm{E}-01$ & $3.61 \mathrm{E}-02$ & $6.93 \mathrm{E}-03$ & $1.76 \mathrm{E}-03$ \\
of ZF & 64-QAM & $5.59 \mathrm{E}-01$ & $1.81 \mathrm{E}-01$ & $3.50 \mathrm{E}-02$ & $8.22 \mathrm{E}-03$ & $2.06 \mathrm{E}-03$ \\
\hline Standard & 256-QAM & $6.42 \mathrm{E}-01$ & $3.73 \mathrm{E}-01$ & $1.65 \mathrm{E}-01$ & $6.18 \mathrm{E}-02$ & $1.90 \mathrm{E}-02$ \\
\cline { 2 - 7 } ZF & 128-QAM & $6.22 \mathrm{E}-01$ & $3.63 \mathrm{E}-01$ & $1.54 \mathrm{E}-01$ & $5.24 \mathrm{E}-02$ & $1.79 \mathrm{E}-02$ \\
& 64-QAM & $6.07 \mathrm{E}-01$ & $3.55 \mathrm{E}-01$ & $1.54 \mathrm{E}-01$ & $5.32 \mathrm{E}-02$ & $1.90 \mathrm{E}-02$ \\
\hline
\end{tabular}

Comparison of detection schemes with type of modulation in $8 \times 8$ uncoded MIMO systems with SER as a performance parameter is as shown in Table 2.

\section{CONCLUSION}

In this article, linear detectors V-BLAST by means of ZF and standard ZF are investigated. They are compared for 8 X 8 uncoded MIMO structure with different modulation schemes. By performing comparison of results it is clear that V-BLAST by means of ZF perform extensively superior as compared to standard ZF based on SER. As the SNR increases SER with V - BLAST by means of $\mathrm{ZF}$ decreases as compared to standard ZF with all modulation schemes of adaptive modulation. Both of these detectors are looked at adaptive modulation schemes with M-QAM where $M=64,128$ and 256. These modulation schemes comparison states that 128-QAM performs superior to staying two. So that use of 128-QAM is possible in LTE with 8 X 8 MIMO with certain tradeoffs. 


\section{REFERENCES}

Ananya. (2014). Performance Evaluation for V-Blast MIMO Systems Under Different Channel Conditions with Various Detection Techniques. International Journal of Advanced Technology \& Engineering Research, 4, 18-22.

de Jong, Y. L. C., \& Willink, T. J. (2005). Detection for MIMO wireless systems. IEEE Transactions on Communications, 53(6), 930-935. doi:10.1109/TCOMM.2005.849638

Ganesan Rajesh, Raajini, Ashoka, Gokuldhev, \& Swetha. (2020). A Multi-objective Routing Optimization Using Swarm Intelligence in IoT Networks, In Intelligent Computing and Innovation on Data Science. Lecture Notes in Networks and Systems (vol. 118). Springer.

Ginis, G., \& Cioffi, J. M. (2001). On the relationship between V-BLAST and GDFE. IEEE Communications Letters, 5(9), 364-366. doi:10.1109/4234.951378

Golden, G. D., Foschini, G. J., Valenzuela, R. A., \& Wolniasky, P. W. (1999). Detection algorithm and initial laboratory results using the V-BLAST space-time communication architecture. Electronics Letters, 35(1), 14-16. doi:10.1049/el:19990058

Hamad Ameen, J. J. (2014). 8 x 8 MIMO System Transmission Characteristics with MC-CDMA Multiplexing For Next Generation Mobile System. Pensee Journal, 76(6), 295-302.

Ilesanmi, B. (2013). Review of Space-time Coded Orthogonal Frequency Division Multiplexing Systems for Wireless Communication. IETE Technical Review, 30(5), 417-426. doi:10.4103/0256-4602.123126

Jiang, Y., Zheng, X., \& Li, J. (2005). Asymptotic analysis of V-BLAST. Proceedings of IEEE GlobeCom, $3886-3890$.

Kühn. (2006). Wireless Communications over MIMO Channels - Applications to CDMA and Multiple Antenna Systems. Wiley.

Loyka, S., \& Gagon, F. (2004). Performance analysis of the V-BLAST algorithm: An analytical approach. IEEE Transactions on Wireless Communications, 3(4), 1326-1337. doi:10.1109/TWC.2004.830853

Nabar, R. U., Boleskei, H., \& Paulraj, A. J. (2005). Diversity and outage performance in Space Time Block Coded Rician MIMO Channels. IEEE Transactions on Wireless Communications, 4(5), 1102-1111. doi:10.1109/ TWC.2005.853835

Nabar, R. U., Paulraj, A. J., Gore, D. A., \& Bolcskei, H. (2004). An overview of MIMO communications-A key to gigabit wireless. Proceedings of the IEEE, 92(2), 198-218. doi:10.1109/JPROC.2003.821915

Paulraj, A., \& Heath, R. J. (2001). Characterization of MIMO Channels for Spatial Multiplexing Systems. IEEE International Conference on Communications, 2, 591-595.

Prasad, N., \& Varanasi, M. K. (2001). Analysis of decision-feedback detection for MIMO Rayleigh fading channels and optimum allocation of transmitter powers and QAM constellations, 39th Annual Allerton Conf. on Commun., Control, and Comp., Univ. of IL.

Seok-Ho, C., Meesue, S., \& Sang-Hyo, K. (2020). Joint Source, Channel, and Space-Time Coding of Progressive Bitstream in MIMO Channels. IEEE Transactions on Communications, 68(4), 2551-2565. doi:10.1109/ TCOMM.2019.2945788

Shweta, S., \& Upena, D. (2014). Enhancing Capability of DVB-H by Three Layer Hierarchical Mapping. IETE Technical Review, 31(2), 162-167. doi:10.1080/02564602.2014.892757

Sinha, N. B., Bera, R., \& Mitra, M. (2010). Capacity and V-BLAST Techniques for MIMO Wireless Channel. Journal of Theoretical and Applied Information Technology, 14(1), 11-21.

Special issue on complexity reduction in MIMO. (2009). IEEE Journal of Selected Topics in Signal Processing, 3,6 .

Telatar, I. E. (1999). Capacity of multi-antenna Gaussian channels. European Transactions onTelecommunications, 10(6), 585-595. doi:10.1002/ett.4460100604 
Wei, Pokhariyal, Sorensen, Kolding, \& Mogensen. (2007). Performance of MIMO with frequency domain packet scheduling in UTRAN LTE downlink. IEEE VTC, 1177-1181.

Wolniosky, P., Foschini, G. J., Golden, G. D., \& Valenzuela, R. A. (1998). V-BLAST: An Architecture for realizing very high data rates over rich scattering wireless channel, URSI International Symposium on Signals, Systems and Electronics, ISSSE 98, 295-300.

Xiao, L., Yue, X., Chao, X., Xia, L., Ping, Y., Li, S., \& Lajos, H. (2018). Compressed-sensing assisted spatial multiplexing aided spatial modulation. IEEE Transactions on Wireless Communications, 17(2), 794-807. doi:10.1109/TWC.2017.2771759

Rajashree Suryawanshi is a researcher at Sathyabama Institute of Science \& Technology, Chennai. Presently working as an Asstistant Professor, Department of E \& TC, Army Institute of Technology, Pune. Her area of interest is LTE, Large MIMO and Swarm Intelligence.

P. Kavipriya is teaching and conducting research at Sathyabama Institute of Science \& Technology Currently B.P. Patil is working as Professor and Principal at Army institute of Technology, Pune. He has received the "Sir Thomas Ward memorial Medal" from the Institution of Engg., Calcutta, for the best paper in E \& T Journal div. for the year 1999-2000. He has published 150 papers at national / international conference \& journals. He has Published 02 books and filed 06 patents. He is Fellow of IETE, Institution of Engineers and Life member of ISTE and Instrument Society of India. His research interest includes wireless communication and signal processing. 